\title{
Theoretical Analysis of Different External trapping potential used in experimental of BEC
}

\author{
Noori.H.N. Al-HASHIMI ${ }^{1}$; Samer K Ghalib \\ Department of Physics; College of Education, University of Basra; Basra; Iraq
}

\begin{abstract}
This paper will focus on theoretical treatment of external trapping potentials which are usually used in experimental that lead to produced Bose-Einstein condensation BEC in ultra cold gases. Several types of trapping potentials such as one dimensional BEC in a harmonic oscillator potential (HOP), one dimensional $B E C$ in a double well potential DWP, and one dimensional BEC in a harmonic plus optical lattice potential HOLP are analyze. These analyses give us the overall view of the region of confinement that the external trapping potentials have employed.
\end{abstract}

Keywords: Laser cooled atom, BEC atom, Trapping

\section{Introduction}

Bose-Einstein condensation BEC has been a widely studied research topic among physicists and applied mathematicians since its first experimental realization of (BEC) in ultra cold atomic gases was initially verified by a sequence of experiments in 1995 by Anderson et al. (vapor of rubidium) and Davis et al. (vapor of sodium) that those atoms were confined in magnetic traps and cooled down to low temperatures at an order of microkelvins [1]. For the detail discussions see also [2-3]. In these verifications, theoretical exploration of characteristic of trapped potential needs a mathematical model describing those potentials which are used experimentally to produce BEC at very low temperatures. Many different shape of Bose-Einstein condensation has been achieved by using different type of trapping potential, for example cigar-shaped BEC which has been considered as an interesting subject especially in the coherent atom optics [4-6]. External parabolic potential in (highly anisotropic) of the axial symmetry has been used to develop BEC see for example [7-12]. In some literatures, many authors investigated the effect of gravitation [13] by adding the gravitational potential as an external interaction. In this paper, we analyze in one dimension the different form of trapping potential which are typically used in experiments of BEC.

\section{Theory}

\section{A) Mathematical background}

Hamiltonian of the quantum field operators $\hat{\psi}(\boldsymbol{r}, t)$ and $\hat{\psi}^{\dagger}(\boldsymbol{r}, t)$ which creates and annihilates a particle at position $r$ at time $t$, can be expressed as

$\widehat{H}=\int \hat{\psi}^{\dagger}(\boldsymbol{r}, t)\left(-\frac{\hbar^{2}}{2 m} \nabla^{2}+V(\boldsymbol{r}, t)\right) \hat{\psi}(\boldsymbol{r}, t) d \boldsymbol{r}+\frac{1}{2} \iint \widehat{\boldsymbol{\psi}}^{\dagger}(\boldsymbol{r}, \boldsymbol{t}) \widehat{\boldsymbol{\psi}}^{\dagger}\left(\boldsymbol{r}^{\prime}, \boldsymbol{t}\right) V_{\text {int }}\left(\boldsymbol{r}^{\prime}-\boldsymbol{r}\right) \widehat{\boldsymbol{\psi}}^{\dagger}(\boldsymbol{r}, \boldsymbol{t}) \widehat{\boldsymbol{\psi}}^{\dagger}\left(\boldsymbol{r}^{\prime}, \boldsymbol{t}\right)(1)$

Where $V(\boldsymbol{r}, t)$ is the external trapping potential and $V_{\text {int }}\left(\boldsymbol{r}^{\prime}-\boldsymbol{r}\right)$ is the two-body interatomic interacting potential.

At zero temperature, all anomalous terms and the non-condensate part can be neglected. This is equivalent to replacing the quantum field $\hat{\psi}(\boldsymbol{r}, t)$ in (1) by the classical field $\psi(\boldsymbol{r}, t)$. It gives rise to a nonlinear Schrodinger equation, the well-known Gross-Pitaevskii equation (GPE),

$i \hbar \frac{\partial \psi(\boldsymbol{r}, t)}{\partial t}=\left[-\frac{\hbar^{2}}{2 m} \nabla^{2}+V(\boldsymbol{r})+g|\psi(\boldsymbol{r}, t)|^{2}\right] \psi(\boldsymbol{r}, t)$

for the Bose-Einstein condensed system. Here The external trapping potential $V(\boldsymbol{r})$ is taken to be timeindependent. The GPE "which is a self-consistent mean field nonlinear Schrodinger equation (NLSE)" was first developed independently by Gross [14] and Pitaevskii [15] in 1961 to describe the vortex structure in superfluid. The macroscopic wave function/order parameter is normalized to the total number of particles in the system, which is conserved over time, i.e.

$\int \mid \psi\left(\boldsymbol{r},\left.t\right|^{2} d \boldsymbol{r}=1\right.$

For ideal (non-interacting) gas, all particles occupy the ground state at $T=0 K$ and $\psi(\boldsymbol{r}, t)$. in the GPE describes the properties of all $N$ particles in the system. For interacting gas, owing to the inter-particle interaction, not all particles condense into the lowest energy state even at zero temperature. This phenomenon is called the quantum depletion. In a weakly interacting dilute atomic vapor, which is the main concern in this thesis, the non-condensate fraction is very small. The mean field theory can be successfully applied and the quantum depletion can be neglected at zero temperature, assuming a pure BEC in the system. 


\section{B) Different external trapping potentials}

In early BEC experiments, quadratic harmonic oscillator well was used to trap the atoms. Recently more advanced and complicated traps have been applied for studying BECs in laboratories [16, 17, 18, 19]. In this section, we will review several typical trapping potentials which are widely used in current experiments.

I. Three-dimensional (3D) harmonic oscillator potential hop [19]:

$$
V_{\text {hop }}(\boldsymbol{r})=V_{\text {hop }}(x)+V_{\text {hop }}(y)+V_{\text {hop }}(z) \quad V_{\text {hop }}(\boldsymbol{r})=\frac{m}{2} \omega_{r}^{2} r^{2}, \quad r=x, y, z
$$

Where, $\omega_{x}, \omega_{y}$, and $\omega_{z}$ are the trapping frequencies in $x$-, $y$-, and $z$-direction respectively.

II. 2D harmonic oscillator + 1D double well potential dwp (Type I) [18]:

$V_{d w p}^{1}(r)=V_{d w p}^{1}(x)+V_{h o p}(y)+V_{h o p}(z) \quad V_{d w p}^{1}(r)=\frac{m}{2} v_{x}^{4}\left(x^{2}-\hat{a}^{2}\right)^{2}$

Where, $\pm \mathrm{a}^{\wedge}$ are the double well centers along the $\mathrm{x}$-axis, $v_{\mathrm{x}}$ is a given constant with physical dimension $1 /[\mathrm{m}$ $\mathrm{s}]^{1 / 2}$.

III.

2D harmonic oscillator $+1 \mathrm{D}$ double well potential dwp (Type II) [20, 21]:

$$
V_{d w p}^{(2)}(r)=V_{d w p}^{2}(x)+V_{h o p}(y)+V_{h o p}(z) \quad V_{d w p}^{(2)}(r)=\frac{m}{2} \omega_{x}^{2}(|x|-\hat{a})^{2}
$$

IV. 3D harmonic oscillator + optical lattice potential optlp [22,23,19]:

$$
V_{\text {hop }}(r)=V_{\text {ho }}(x)+V_{\text {opt }}(x)+V_{\text {opt }}(y)+V_{\text {opt }}(z) \quad V_{\text {opt }}(\tau)=S_{\tau} E_{\tau} \sin ^{2}\left(\hat{q}_{\tau} \tau\right)(8)
$$

where $\hat{q}_{\tau}=2 \pi / \lambda_{\tau}$ is the angular frequency of the laser beam, with wavelength $\lambda_{\tau}$, that creates the stationary 1D periodic lattice, $\mathrm{E}_{\tau}=\left(\hbar^{2} \hat{q}_{\tau}^{2}\right) / 2 \mathrm{~m}$ is the recoil energy, and $\mathrm{S}_{\tau}$ is a dimensionless parameter characterizing the intensity of the laser beam. The optical lattice potential has periodicity $\mathrm{T}_{\tau}=\pi / \hat{q}_{\tau}=\lambda_{\tau} / 2$ along the $\tau$-axis $(\tau=\mathrm{x} ; \mathrm{y}$; z).

V. 3D box potential [19]:

$V_{\text {box }}(x)=\left\{\begin{array}{cc}0 & 0<x, y, z<L \\ \infty & \text { otherwise }\end{array}\right.$

where $\mathrm{L}$ is the length of the box.

\section{C) Dimensionless External Potential:}

The choices for the scaling parameters $t_{0}$ and $x_{0}$, the dimensionless potential $V(r)$ with $\gamma_{y}=t_{0} \omega_{y}$ and $\gamma_{z}=$ $t_{0} \omega_{z}$, the energy unit $E_{0}=\hbar / t_{0}=\hbar^{2} / m r_{0}^{2}$, and the interaction parameter $\beta=4 \pi a_{s} N / r_{0}$ for different external

trapping potentials are given below:

I. $\quad t_{0}=\frac{1}{\omega_{r}}, r_{0}=\sqrt{\frac{\hbar}{m \omega_{r}}} \quad V(r)=\frac{1}{2}\left(x^{2}+\gamma_{y}^{2} y^{2}+\gamma_{z}^{2} z^{2}\right)$,

II. $2 \mathrm{D}$ harmonic oscillator $+1 \mathrm{D}$ double well potential (type I):

$$
t_{0}=\left(\frac{m}{\hbar v_{r}^{4}}\right)^{1 / 3}, r_{0}=\left(\frac{\hbar}{m v_{r}^{2}}\right)^{1 / 3}, a=\frac{\hat{a}}{r_{0}}, V(r)=\frac{1}{2}\left[\left(x^{2}-a^{2}\right)^{2}+\gamma_{y}^{2} y^{2}+\gamma_{z}^{2} z^{2}\right]
$$

III. 2D harmonic oscillator $+1 \mathrm{D}$ double well potential (type II):

$$
t_{0}=\frac{1}{\omega_{r}}, r_{0}=\sqrt{\frac{\hbar}{m \omega_{r}}}, a=\frac{\hat{a}}{r_{0}}, \quad V(r)=\frac{1}{2}\left((|r|-a \mid)^{2}+\gamma_{y}^{2} y^{2}+\gamma_{z}^{2} z^{2}\right)
$$

IV. 3D harmonic oscillator + optical lattice potential:

$$
\begin{aligned}
& t_{0}=\frac{1}{\omega_{r}}, \quad r_{0}=\sqrt{\frac{\hbar}{m \omega_{r}}}, \quad k_{r}=\frac{2 \pi^{2} r_{0}^{2} S_{\tau}}{\lambda_{\tau}^{2}}, \quad q_{\tau}=\frac{2 \pi r_{0}}{\lambda_{\tau}} \quad \tau=x, y, z \\
& V(r)=\frac{1}{2}\left(x^{2}+\gamma_{y}^{2} y^{2}+\gamma_{z}^{2} z^{2}\right)+k_{x} \sin ^{2}\left(q_{x} x\right)+k_{x y} \sin ^{2}\left(q_{y} y\right)+k_{z} \sin ^{2}\left(q_{z} z\right) .
\end{aligned}
$$

V. 3D box potential:

$$
r_{0}=\frac{m L^{2}}{\hbar}, \quad r_{0}=L \quad V(x)=\left\{\begin{array}{rr}
0 & 0<x, y, z<1 \\
\infty & \text { otherwise }
\end{array}\right.
$$

\section{Result And Discussion:}

For simplicity we shall discuss the trapping potential in one dimension as follow:

a) Under external potentials I-IV For a 1D BEC in a harmonic oscillator potential, $V(x)=\frac{1}{2}\left(\gamma_{x}^{2} x^{2}\right), \gamma_{x}>0$ the shape of this potential for different values of $\boldsymbol{\gamma}_{\boldsymbol{x}}(0.5,1,1.5,2)$ is shown in figure (1). The influences of $\gamma_{x}$ on the shape of harmonic oscillator potential are clear were the potential will broadening as $\gamma_{x}$ decrease. More study of the harmonic oscillator potential is carried out at specific point along the axis of propagation, these points are (2., 4., 6., 8., 10.) as shown in figure (2). One can conclude from this figure that the 1D HOP depends on both the position along the propagation axis and the value of $\gamma_{x}$. The 1D 
HOP is nearly a straight line at low value of $\mathrm{x}$-axis, and the shape of this potential become parabola as the axis of propagation increase from 2 ., to 10 .

b) The Double well potential Type I

$$
V(x)=\frac{1}{2}\left(\gamma_{x}^{4}\left(x^{2}-a^{2}\right)^{2}\right), \gamma>0, \quad a \geq 0
$$

where $\gamma>0$ measures the height of the well and $\pm a$ are the centers of the double well

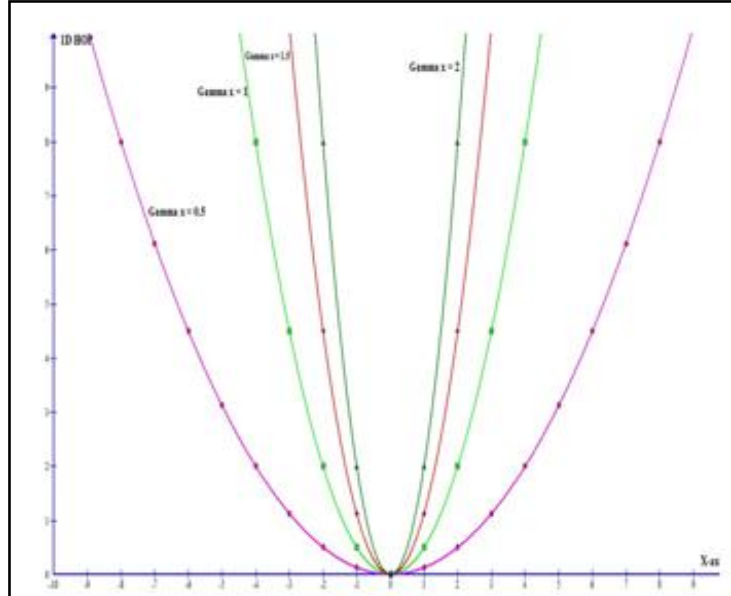

Figure (1) one dimensional harmonic oscillator potential used in experimental of Bose Einstein Condensation

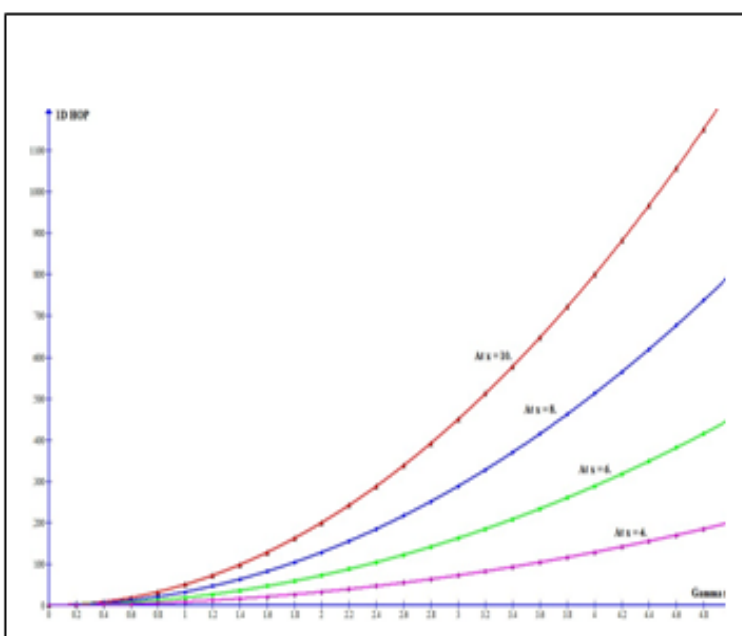

Figure (2) One dimensional harmonic oscillator potential used in experimental of Bose Einstein Condensation as a function of Gammax

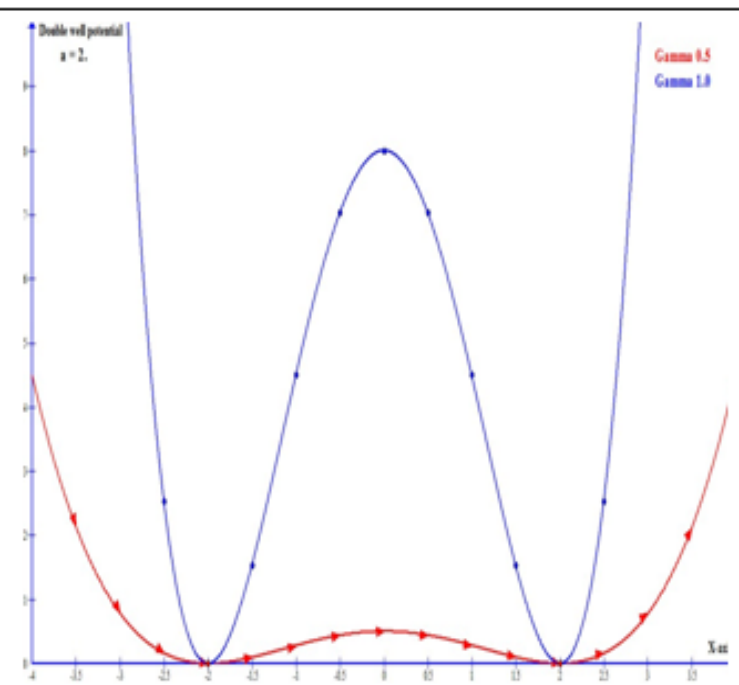

Figure (3) Double well potential used in experimental of Bose Einstein Condensation as a function of Gamma x

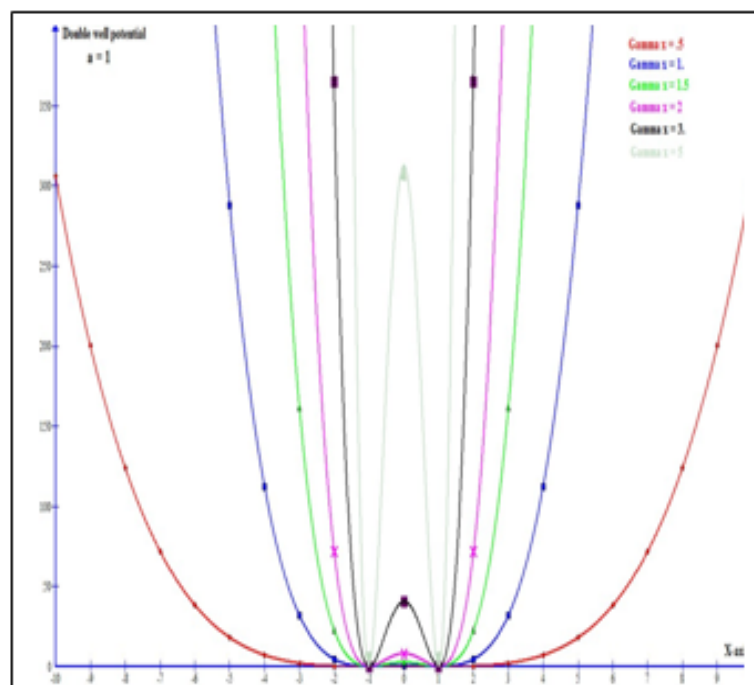

Figure (4) Double well potential used in experimental of Bose Einstein Condensation as a function of Gamma X 

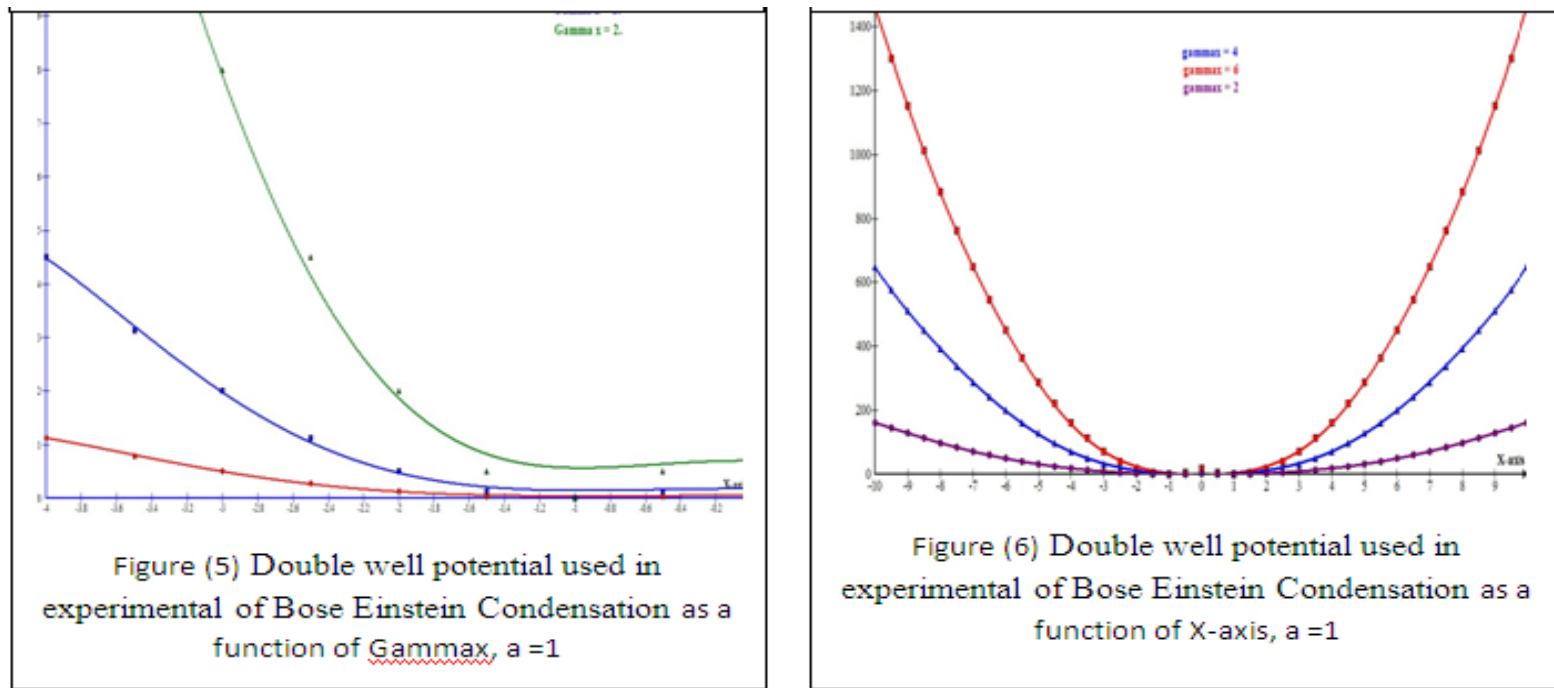

Figure (6) Double well potential used in experimental of Bose Einstein Condensation as a function of $\mathrm{X}$-axis, $\mathrm{a}=1$

This type of potentials are used for the dynamics of attractively interacting condensate in the double well it is known that they exhibit the self-trapping property, in spite of the symmetry of the trap. Figure (3) the centre of the Double well potential is \pm 2 and the high of the well are taken to be 0.5 and 1.0 respectively. Figure (4) are plotted with centre at \pm 1 and high of the well are taken to be $0.5,1.0,2.0,3.0$, and 5.0.

[Remark] In physics literature [24, 25], another type of double well potential, Type II is used $V(x)=\frac{1}{2}\left(\gamma_{x}^{2}(|x|-a)^{2}\right), \gamma>0, a \geq 0$

In figures (5-6) shown type II of double well potential are plot as a function of propagation axis for centre of the well at \pm 1 and for different values of potential high.

c) Optical lattice potential: For a $1 \mathrm{D} \mathrm{BEC}$ in a harmonic plus optical lattice potential,

$V(x)=\frac{1}{2}\left(\gamma_{x}^{2} x^{2}\right)+k_{x} \sin ^{2}\left(q_{x} x\right), k_{x}=25, q_{x}=\pi / 4$

Figure (7) shows the OLP as a function of $\mathrm{x}$-axis for $\gamma_{\mathrm{x}}=1$ and $\mathrm{k}_{\mathrm{x}}=25$ where $\mathrm{q}_{\mathrm{x}}$ take the values $(\pi / 4, \pi / 3, \pi / 6$, and $\pi / 12$ ). One can conclude from this figures that $\mathrm{q}_{\mathrm{x}}$ play a major part in developing the trapping potential used in experimental of BEC. The effect of $\mathrm{q}_{\mathrm{x}}$ becomes more clearly in figure (8) where the values of $\mathrm{k}_{\mathrm{x}}$ in this case vary from 5-30. The relation between OPL and $k_{x}$ are linear as shown in figure (9) for different values of $q_{x}$. As a conclusion to this work one can say that the trapping potential used in experimental of BEC in term of shape and values can be understood from the above figures. To developed a comprehensive pictures about these potential more Analysis should be carried out in 2D and 3D.
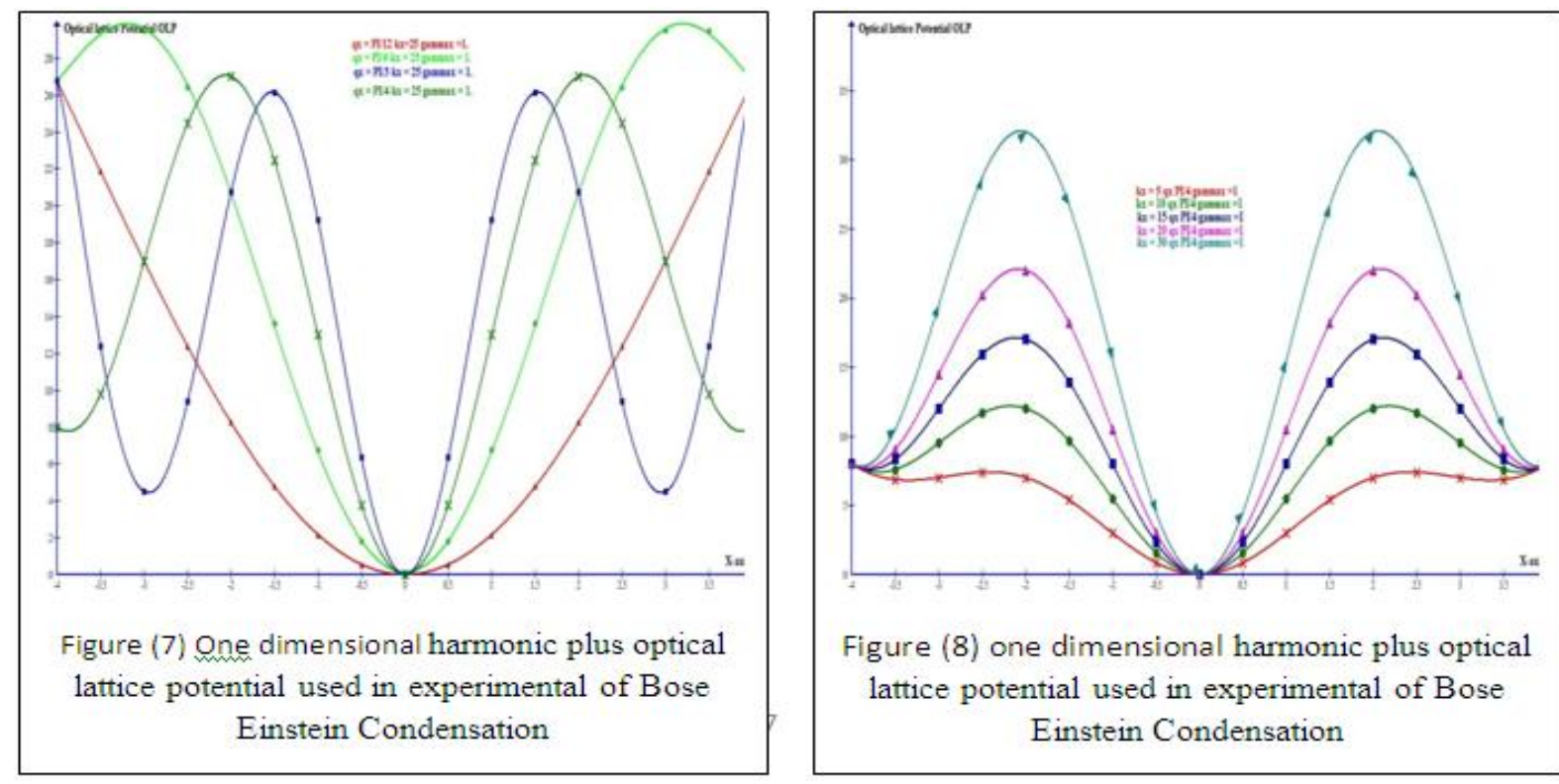


\section{Refrences}

[1] F. Dalfovo, S. Giorgini, L.P. Pitaevskii, S. Stringari, Rev. Mod. Phys. 71 (1999) 463.

[2] M.H. Anderson, J.R. Ensher, M.R. Matthews, C.E. Wieman, E.A. Cornell, Science 269 (1995) 198.

[3] K.B. Davis, M.O. Mewes, M.R. Andrews, N.J. van Druten, D.S. Durfee, D.M. Kurn, W. Ketterle, Phys. Rev. Lett. 75 (1995) 3969.

[4] W. Ketterle, Rev. Mod. Phys. 74 (2002) 1131.

[5] Y. Shin, C. Sanner, G.B. Jo, T.A. Pasquini, M. Saba, W. Ketterle, D.E. Pritchard, Phys. Rev. A 72 (2005) 021604, condmat/0506464.

[6] Y.J. Wang, D.Z. Anderson, V.M. Bright, E.A. Cornell, Q. Diot, T. Kishimoto, M. Prentiss, R.A. Saravanan, S.R. Segal, S. Wu, Phys. Rev. Lett. 94 (2005) 090405.

[7] V.M. Perez-Garcia, H. Michinel, H. Herrero, Phys. Rev. A 57 (1998) 3837.

[8] E.A. Ostrovskaya, Y.S. Kivshar, M. Lisak, B. Hall, F. Cattani, D. Anderson, Phys. Rev. A 61 (2000) 031601

[9] Y.S. Kivshar, T.J. Alexander, S.K. Turitsyn, Phys. Lett. A 278 (2001) 225.

[10] R.A. Duine, H.T.C. Stoof, Phys. Rev. A 65 (2001) 013603.

[11] U. Al Khawaja, H.T.C. Stoof, R.G. Hulet, K.E. Strecker, G.B. Partridge, Phys. Rev. Lett. 89 (2002) 200404.

[12] Jian-Chun Jing and Biao Li, CHINESE JOURNAL OF PHYSICS VOL. 50, NO. 3 June 2012.

[13] Th. Busch, M. Kohl, T. Esslinger, K. Molmer, Phys. Rev. A 65 (2002) 043615.

[14] E. P. Gross. Structure of a quantized vortex in boson systems. Nuovo. Cimento., 20, 454, 1961

[15] L. P. Pitaevskii. Vortex lines in an imperfect Bose gas. Soviet Phys. JETP, 13, 451, 1961.

[16] J. Bronski, L. Carr, B. Deconinck, J. Kutz, and K. Promislow. Stability of repulsive Bose- Einstein condensates in a periodic potential. Phys. Rev. E, 63, 036612, 2001.

[17] L. D. Carr, C. W. Clark, and W. Reinhardt. Stationary solutions of the one-dimensional nonlinear Schroedinger equation: I. case of repulsive nonlinearity. Phys. Rev. A, 62, 063610, 2000.

[18] G. Milburn, J. Corney, E. Wright, and D. Walls. Quantum dynamics of an atomic Bose-Einstein condensate in a double-well potential. Phys. Rev. A, 55, 4318, 1997.

[19] L. Pitaevskii and S. Stringari. Bose-Einstein condensation. Clarendon Press, 2003.

[20] P. Capuzzi and S. Hernandez. Bose-Einstein condensation in harmonic double wells. Phys. Rev. A, 59, 1488, 1999.

[21] M. Holthaus. Towards coherent control of a Bose-Einstein condensate in a double well. Phys. Rev. A, 64, 2001.

[22] S. Adhikari and P. Muruganandam. Mean- ${ }^{-}$eld model for the interference of matter-waves from a three-dimensional optical trap. Phys. Lett. A, 310, 229, 2003.

[23] D.-I. Choi and Q. Niu. Bose-Einstein condensates in an optical latice. Phys. Rev. Lett., 82, 2022, 1999.

[24] P. Capuzzi and S. Hernandez. Bose-Einstein condensation in harmonic double wells. Phys. Rev. A, 59, 1488, 1999.

[25] M. Holthaus. Towards coherent control of a Bose-Einstein condensate in a double well. Phys. Rev. A, 64, 2001. 